\title{
Journal of Orthopedic Research and Therapy
}

\section{Polyacrylamide Hydrogel Injection for Knee Osteoarthritis: A 6 Months Prospective Study}

\section{Henning Bliddal $^{1 *}$, Anders Overgaard ${ }^{1}$, Andreas Hartkopp ${ }^{2}$, Jannie Beier ${ }^{3}$, Philip G Conaghan ${ }^{4}$ and Marius Henriksen $^{1}$}

${ }^{1}$ The Parker Institute, Bispebjerg-Frederiksberg Hospital, University of Copenhagen, Copenhagen, Denmark

${ }^{2}$ A2 Rheumatology and Sports Medicine, Hillerød, Denmark

${ }^{3}$ Gigtdoktor, Odense, Denmark

${ }^{4}$ Leeds Institute of Rheumatic and Musculoskeletal Medicine, University of Leeds \& NIHR Leeds Biomedical Research Centre, United Kingdom

"Corresponding author: Henning Bliddal, The Parker Institute, Bispebjerg-Frederiksberg Hospital, University of Copenhagen, Copenhagen, Denmark

Citation: Bliddal H, Overgaard A, Hartkopp A, Beier J, Conaghan PG, et al. (2021) Polyacrylamide Hydrogel Injection for Knee Osteoarthritis: A 6 Months Prospective Study. J Orthop Res Ther 6: 1188. DOI: 10.29011/2575-8241.001188

Received Date: 11 March, 2021; Accepted Date: 22 March, 2021; Published Date: 25 March, 2021

\begin{abstract}
Objective: Intra-Articular (IA) injection of polyacrylamide hydrogel (PAAG) is a possible treatment for symptomatic Osteoarthritis (OA) of the knee. This study evaluated the efficacy and safety of a single injection of $6 \mathrm{ml}$ intra-articular PAAG over 26 weeks.

Methods: Open-label study in patients with symptomatic and radiographically confirmed knee OA . Primary outcome was change in WOMAC pain after 13 weeks. Secondary outcomes were WOMAC stiffness and function subscales, Patient Global Assessment of disease impact (PGA) and proportion of OMERACT-OARSI responders. Follow-up time points were 4, 13 and 26 weeks.

Results: 49 patients ( 31 females) received PAAG, with 48 patients completing the 13 and 46 the 26 weeks assessments. Mean change in WOMAC pain after 13 weeks was -18.3 points [95\% CI-23.4 to -13.3 ]; $\mathrm{P}<.0001$ and at 26 weeks -20.8 points [95\% CI -26.3 to -15.3]; $\mathrm{P}<0.0001$ with similar benefits for WOMAC stiffness, physical function, and PGA. After 13 weeks $64.6 \%$ were OMERACT-OARSI responders and this was maintained at 26 weeks.. During the 13 weeks, 18 patients reported 23 adverse events, 13 of which were related to PAAG, none severe. Two serious adverse events, atrial fibrillation and gastrointestinal pain, were assessed as 'not related' to PAAG.

Conclusions: PAAG can be delivered in a single $6 \mathrm{ml}$ injection and this non-randomized trial in patients with knee OA demonstrated beneficial clinical effects at 13 and 26 weeks. No serious adverse events were seen with PAAG. These encouraging results need to be confirmed in controlled studies.
\end{abstract}

Keywords: Intra-articular treatment; Osteoarthritis

\section{Background}

Osteoarthritis (OA) is the most common arthritis, characterised by pain and physical disability [1]. More than $10 \%$ of persons 55 years of age or older have symptomatic OA, primarily involving the knees [2]. Although several treatment options for $\mathrm{OA}$ in the knee are available, non-pharmacological treatments (e.g. exercise, weight loss if overweight) are often underutilised, analgesics have limited benefits, and joint replacement surgery is limited to end-stage disease. New treatment options are therefore warranted in order to improve the care of people with OA. Recently,
Intra-Articular (IA) injection of polyacrylamide gel (PAAG) has been suggested as a possible treatment for symptomatic OA. PAAG is a proprietary $2.5 \%$ cross-linked polyacrylamide hydrogel, manufactured by Contura International A/S. PAAG contains $2.5 \%$ polyacrylamide and $97.5 \%$ non-pyrogenic water, with a unique molecular structure that allows normal water exchange with the surrounding tissue without losing shape. PAAG is biocompatible, non-absorbable, non-biodegradable, and structurally stable. For more than 20 years, PAAG has been used for various indications such as bulking for stress urinary incontinence and soft tissue augmentation. PAAG is classified as a Class IIb device under the European Council Directive 93/42/EEC on medical devices. 


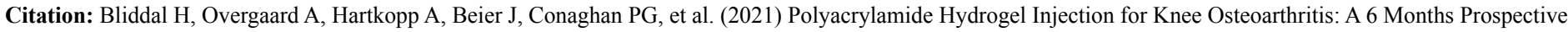
Study. J Orthop Res Ther 6: 1188. DOI: 10.29011/2575-8241.001188

PAAG has been extensively tested in horses with OA with pain relief observed within a month, with the majority of the horses no longer being lame after 24-months [3]. An observational study in patients with knee OA reported that IA PAAG delivered as two injections of $3 \mathrm{ml}$ separated by 1 month provided symptomatic relief up to 13 months [4]. If $6 \mathrm{ml}$ can be delivered in one treatment session, this would improve patient acceptability, feasibility of treatment and reduce the risk of potential side effects related to repeated injections. The objectives of this study were to evaluate the efficacy and safety of one injection of $6 \mathrm{ml}$ IA PAAG on knee pain in patients with moderate to severe knee OA.

\section{Methods}

This study was an open label, 26-week observational study of the efficacy and safety of intra-articular (IA) injection of PAAG with data collected from August 2019 to April 2020 at three clinical sites in Denmark. The protocol for this study was approved by the local Health Research Ethics committee (ref.no: H-19031685), the Danish Health authorities, and was registered at www.clinicaltrials. gov (NCT04179552) before any study related activities. Patients gave informed consent prior to participation, and the study was conducted according to the principles of good clinical practice. Inclusion criteria were: adults with a clinical diagnosis of knee OA according to the American College of Rheumatology, definite radiographic OA in the target knee (Kellgren-Lawrence grade 2-4) (locally read), score of $\geq 2$ (on 0-4 scale) on the Western Ontario and McMaster Universities Osteoarthritis Index (WOMAC) question related to pain while walking on a flat surface, a body mass index (BMI) $<35 \mathrm{~kg} / \mathrm{m}^{2}$, and, if patients were taking oral analgesics, they needed to have been on a stable dose for at least 4 weeks prior to inclusion. Exclusion criteria included: previous IA PAAG treatment, IA hyaluronic acid or its derivatives in target knee within prior 6 months, IA corticosteroids within 3 months, skin disease in the injection area, and surgery in the treatment knee within 6 months.

The patients received one treatment of $6 \mathrm{ml}$ PAAG and attended clinical follow-up visits after 4, 13, and 26 weeks.

\section{Treatments Administered}

PAAG was provided in sterile, pre-filled $1 \mathrm{ml}$ sealed syringes to be injected IA with a sterile $21 \mathrm{G} \times 2$-inch $(0.8 \times 50$ $\mathrm{mm})$ needle. Each patient received one IA injection of PAAG $(6 \mathrm{ml})$ under ultrasound guidance to ensure proper placement of the bolus inside the joint cavity. Prior to the injection, prophylactic antibiotics were given and topical anaesthetics were applied. Once the needle was inserted, the prefilled $1 \mathrm{ml}$ PAAG syringes were changed via the Luer lock system to reach a total of $6 \mathrm{ml}$ of PAAG with the needle remaining in place. Analgesic treatment with nonsteroidal anti-inflammatory drugs (NSAIDs) and paracetamol was allowed during the trial, but not exceeding the recommended dosage (e.g. paracetamol up to $4000 \mathrm{mg}$ /day and ibuprofen 1200 $\mathrm{mg}$ /day), but not allowed within 48 hours prior to a study visit. Non-pharmacological therapy was allowed if kept stable during the study. All concomitant therapies and treatments were recorded.

\section{Outcomes}

The primary outcome measure was change from baseline in the pain subscale of the WOMAC at 13 weeks. Secondary outcome measures were changes in baseline in the physical function and stiffness subscales of WOMAC, Patient's Global Assessment (PGA) of OA impact, and the proportion of positive responders to the OMERACT-OARSI response criteria [5]. Data were collected at baseline, and after 4, 13, and 26 weeks. In this study, the Danish WOMAC was used in the 5-point Likert format with scores ranging from 0 (indicating no pain, stiffness, or difficulty) to 4 (indicating extreme pain, stiffness, or difficulty). The three WOMAC subscale scores were normalised to a 0-100 scale with 0 indicating best outcome and 100 indicating worst outcome. The PGA was based on responses to the question "How much does the knee osteoarthritis (treatment knee) as a whole affect your life at present?" indicated on a $100 \mathrm{~mm}$ Visual Analogue Scale (VAS) with anchors $0=$ "Not at all" and $100=$ "The worst imaginable". OMERACT-OARSI response was defined as either (1) improvement in WOMAC pain or physical function $\geq 50 \%$ and an absolute change $\geq 20$ normalised units (0-100); or (2) $\geq 20 \%$ improvement and an absolute change $\geq 10$ points two of the three categories: WOMAC pain, WOMAC physical function, and PGA

\section{Statistical Methods}

The sample size was calculated to detect a change of at least 12 points on the WOMAC pain subscale (0-100 points) using a one-sample t-test and a statistical significance level of 0.05 , assuming a common standard deviation of 22 points. To obtain $90 \%$ power a sample size of 38 was required. The analyses of the primary outcome were performed by using a repeated measures mixed linear model with a restricted maximum likelihood based approach and carried out on the full analyses set population defined as patients who received study treatment as per the protocol and who had a valid recording of the WOMAC pain subscale at baseline. Missing data were not imputed but handled implicitly by the repeated measures mixed linear model. The analyses of the secondary outcomes were performed using the same statistical model as for the primary outcome applied to the same population defined as all allocated subjects who received study treatment. The proportion of positive responders to the OMERACT-OARSI criteria at time points 4 weeks, 13 weeks, and 26 weeks were summarised including exact Clopper-Pearson 95\% confidence intervals. Internationally applicable estimates of minimal clinically important improvements (MCII) of the WOMAC scores on the $0-100$ scale have been suggested for individuals with knee OA in a 4-week study of nonsteroidal anti-inflammatory drugs [6]. For the WOMAC pain subscale the MCII is 9 points; for the stiffness 


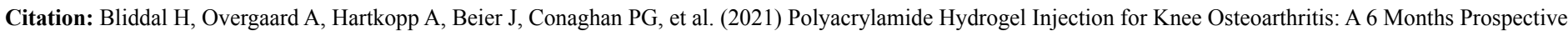
Study. J Orthop Res Ther 6: 1188. DOI: 10.29011/2575-8241.001188

subscale MCII it is 7 points; and for the function subscale MCII it is 6 points. For transformed WOMAC pain, stiffness and physical function subscales the proportion of subjects fulfilling the MCII criteria at week 26 were summarised including exact ClopperPearson $95 \%$ confidence intervals.

\section{Results}

50 subjects (31 females, mean age 70 SD 9 years) were screened and 49 enrolled in the study (Table 1). One subject received only approximately $5 \mathrm{ml}$ of PAAG due to a user error; thus, the full analysis set consisted of 48 subjects. All 48 subjects completed the 4 and 13 week assessments, and 46 completed the week 26 assessment.

Table 2 shows the results from the main analyses. At the 13 weeks follow-up there was a decrease in the WOMAC pain score of -18.3 points $(95 \% \mathrm{CI}-23.3$ to $-13.3 ; \mathrm{P}<.0001)$. This was further improved at 26 weeks to -20.8 (95\% CI -26.2 to -15.3 ; $\mathrm{P}<.0001)$. Similar results were found for the WOMAC stiffness and function subscales, which improved at the 4, 13 and 26 week assessments. WOMAC pain, stiffness and function subscales data at the 26-week assessment are provided in Figure 1. At 4, 13 and 26 weeks, the PGA had decreased by $-13.4 \mathrm{~mm}(95 \% \mathrm{CI}-19.5$ to $-7.3 ; \mathrm{P}<.0001) ;-18.7 \mathrm{~mm}(95 \% \mathrm{CI}-25.3$ to $-12.1 ; \mathrm{P}<.0001)$, and $-15.4 \mathrm{~mm}(-23.5$ to $-7.6 ; \mathrm{P}=0.0003)$, respectively.

\begin{tabular}{|c|c|}
\hline & $\begin{array}{c}\text { PAAG-OA } \\
\text { N (\%) }\end{array}$ \\
\hline Screened subjects & 50 \\
\hline Screening failures & 1 \\
\hline & \\
\hline ITT analysis set & $49(100.0)$ \\
\hline Full analysis set & $48(98.0)$ \\
\hline Per protocol analysis set & $41(83.7)$ \\
\hline & $3(6.1)$ \\
\hline Withdrawn until 26 weeks & $1(33.3)$ \\
\hline Adverse event & $2(66.7)$ \\
\hline Withdrawal by subject & \\
\hline \multicolumn{2}{|c|}{} \\
\hline Completers of 26 weeks & $46(93.9)$ \\
\hline N: Number of subjects; \%: Percentage of subjects \\
\hline
\end{tabular}

Table 1: Subject disposition.

\begin{tabular}{|c|c|c|c|c|c|c|}
\hline & \multicolumn{2}{|c|}{ WOMAC Pain subscale } & \multicolumn{2}{|c|}{ WOMAC Stiffness subscale } & \multicolumn{2}{|c|}{ WOMAC Physical function subscale } \\
\hline & Actual values & $\begin{array}{c}\text { Change from } \\
\text { baseline }\end{array}$ & Actual values & $\begin{array}{c}\text { Change from } \\
\text { baseline }\end{array}$ & Actual values & $\begin{array}{c}\text { Change from } \\
\text { baseline }\end{array}$ \\
\hline $\begin{array}{l}\text { Full analysis set } \\
(\mathrm{N}, \%)\end{array}$ & $49(100.0)$ & & & & & \\
\hline \multicolumn{7}{|l|}{ Baseline } \\
\hline $\mathrm{N}$ & 49 & & 49 & & 49 & \\
\hline Mean (SD) & $50.3(11.8)$ & & $55.6(17.5)$ & & $46.6(16.1)$ & \\
\hline Median & 50.0 & & 62.5 & & 45.6 & \\
\hline Min - Max & $20-75$ & & $0-88$ & & $9-87$ & \\
\hline \multicolumn{7}{|l|}{ Week 4} \\
\hline $\mathrm{N}$ & 49 & 49 & 49 & 49 & 49 & 49 \\
\hline Mean (SD) & $34.9(16.4)$ & $-15.4(15.3)$ & $44.1(20.6)$ & $-11.5(17.3)$ & $33.4(16.7)$ & $-13.2(13.8)$ \\
\hline LSMean & -15.4 & & -11.4 & & -13.2 & \\
\hline $95 \% \mathrm{CI}$ & $(-19.7 ;-11.2)$ & & $(-16.2 ;-6.6)$ & & $(-16.9 ;-9.6)$ & \\
\hline Median & 35.0 & -15.0 & 50.0 & -12.5 & 35.3 & -10.3 \\
\hline
\end{tabular}


Citation: Bliddal H, Overgaard A, Hartkopp A, Beier J, Conaghan PG, et al. (2021) Polyacrylamide Hydrogel Injection for Knee Osteoarthritis: A 6 Months Prospective Study. J Orthop Res Ther 6: 1188. DOI: 10.29011/2575-8241.001188

\begin{tabular}{|c|c|c|c|c|c|c|}
\hline & \multicolumn{2}{|c|}{ WOMAC Pain subscale } & \multicolumn{2}{|c|}{ WOMAC Stiffness subscale } & \multicolumn{2}{|c|}{ WOMAC Physical function subscale } \\
\hline & Actual values & $\begin{array}{c}\text { Change from } \\
\text { baseline }\end{array}$ & Actual values & $\begin{array}{c}\text { Change from } \\
\text { baseline }\end{array}$ & Actual values & $\begin{array}{c}\text { Change from } \\
\text { baseline }\end{array}$ \\
\hline Min - Max & $0-75$ & $-50-15$ & $0-88$ & $-50-38$ & $1-63$ & $-38-22$ \\
\hline \multicolumn{7}{|l|}{ Week 13} \\
\hline $\mathrm{N}$ & 48 & 48 & 48 & 48 & 48 & 48 \\
\hline Mean (SD) & $32.1(18.2)$ & $-18.0(18.5)$ & $34.6(21.0)$ & $-20.8(19.9)$ & $29.4(18.0)$ & $-17.1(16.1)$ \\
\hline LSMean & -18.3 & & -21.0 & & -17.2 & \\
\hline $95 \%$ CI & $(-23.4 ;-13.3)$ & & $(-26.4 ;-15.7)$ & & $(-21.5 ;-12.9)$ & \\
\hline Median & 35.0 & -20.0 & 25.0 & -25.0 & 28.7 & -19.8 \\
\hline Min - Max & $0-75$ & $-55-35$ & $0-75$ & $-63-38$ & $0-60$ & $-44-25$ \\
\hline \multicolumn{7}{|l|}{ Week 26} \\
\hline $\mathrm{N}$ & 46 & 46 & 46 & 46 & 46 & 46 \\
\hline Mean (SD) & $28.9(18.6)$ & $-21.7(19.6)$ & $37.2(21.5)$ & $-17.9(21.2)$ & $28.6(20.3)$ & $-18.4(17.0)$ \\
\hline LSMean & -20.8 & & -17.5 & & -18.0 & \\
\hline $95 \%$ CI & $(-26.3 ;-15.3)$ & & $(-23.3 ;-11.8)$ & & $(-23.0 ;-13.1)$ & \\
\hline Median & 30.0 & -22.5 & 31.3 & -18.8 & 28.7 & -20.6 \\
\hline Min - Max & $0-70$ & $-60-25$ & $0-75$ & $-50-50$ & $0-62$ & $-47-21$ \\
\hline
\end{tabular}

Table 2: Summary of transformed WOMAC pain subscale (0-100) - 26 weeks ITT analysis set.

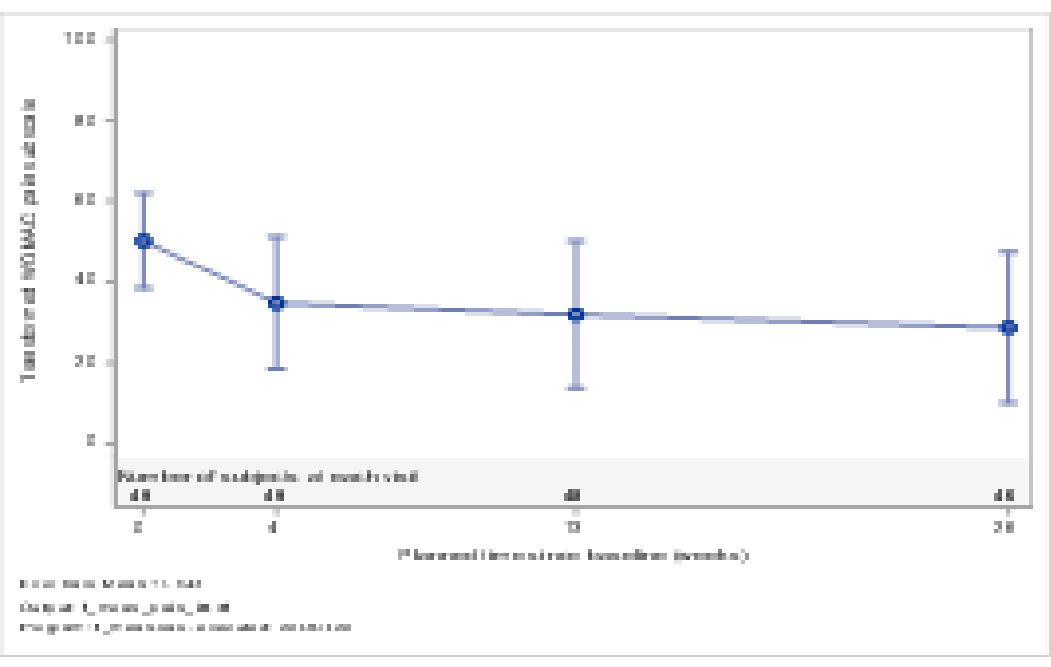

Figure 1: Mean plot of transformed WOMAC pain subscale (0-100) - 26 weeks - ITT analysis set. 


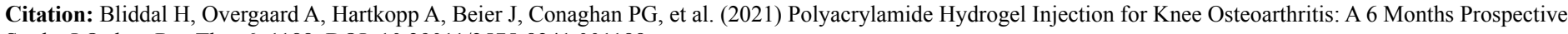
Study. J Orthop Res Ther 6: 1188. DOI: 10.29011/2575-8241.001188

The proportion of OMERACT-OARSI responders at the 4, 13 and 26 week assessments were 53.1\%; (95\% CI 38.3 to 67.5); 64.6\% (95\% CI 49.5 to; 77.8), and 67.4\% (95\% CI 51.98 to 80.47), respectively. The proportion of patients with a response larger than the MCII in the transformed WOMAC subscales at week 26 was highest for the WOMAC pain subscale (78\%) but was greater than $70 \%$ for all 3 subscales (Table 3).

\begin{tabular}{|c|c|c|c|}
\hline & $\mathbf{N}$ & n (\%) & $(95 \% \mathrm{CI})$ \\
\hline ITT analysis set & 49 & & \\
\hline $\begin{array}{c}\text { Transformed WOMAC pain } \\
\text { subscale }\end{array}$ & 46 & $36(78.3)$ & $(63.6 ; 89.1)$ \\
\hline $\begin{array}{c}\text { Transformed WOMAC stiffness } \\
\text { subscale }\end{array}$ & 46 & $34(73.9)$ & $(58.9 ; 85.7)$ \\
\hline $\begin{array}{c}\text { Transformed WOMAC physical } \\
\text { function subscale }\end{array}$ & 46 & $34(73.9)$ & $(58.9 ; 85.7)$ \\
\hline \multicolumn{4}{|c|}{$\begin{array}{l}\text { N: Number of subjects, n: number of subjects achieving the minimal clinically important improvements (MCII) on the WOMAC subscales } \\
\text { MCII: Change in WOMAC pain } \leq-9 \text {, Change in WOMAC stiffness } \leq-7 \text {, Change in WOMAC physical function } \leq-6 \\
\text { CI: Clopper-Pearson exact confidence interval for the proportion }\end{array}$} \\
\hline
\end{tabular}

Table 3: Summary of minimal clinically important improvements for WOMAC subscales - 26 weeks.

Safety

Twenty-one participants reported a total of 31 adverse events (AEs) during the 26-week study period, of which 14 events were assessed to be related to PAAG (Table 4). 12 events (in 11 patients) were musculoskeletal and connective tissue disorders with 11 events being in the treated knee. The most frequently treatment related AEs were arthralgia (7 events), joint swelling (3 events) and synovial cysts ( 2 events). Almost all treatment related AEs were mild, with 3 classified as moderate (Table 5). Two events were reported as serious adverse events (SAEs): 1 hospitalisation with atrial fibrillation and 1 hospitalisation with abdominal pain; neither were assessed as related to the device. No deaths were reported, and no AE led to withdrawal from the study (Figures 2,3).

\begin{tabular}{|c|c|}
\hline & $\begin{array}{c}\text { PAAG-OA } \\
\text { N }(\%) \mathbf{E} \\
\end{array}$ \\
\hline ITT analysis set & 49 \\
\hline Adverse events (AEs) & $20(40.8) 27$ \\
\hline Serious AEs & $2(4.1) 2$ \\
\hline Non-serious AEs & $20(40.8) 25$ \\
\hline Treatment emergent AEs (TEAEs) & $20(40.8) 27$ \\
\hline Non-treatment emergent AEs & 0 \\
\hline Adverse device effects (ADEs) & $12(24.5) 15$ \\
\hline Serious ADEs (SADEs) & 0 \\
\hline AEs leading to withdrawal from study & 0 \\
\hline
\end{tabular}


Citation: Bliddal H, Overgaard A, Hartkopp A, Beier J, Conaghan PG, et al. (2021) Polyacrylamide Hydrogel Injection for Knee Osteoarthritis: A 6 Months Prospective Study. J Orthop Res Ther 6: 1188. DOI: 10.29011/2575-8241.001188

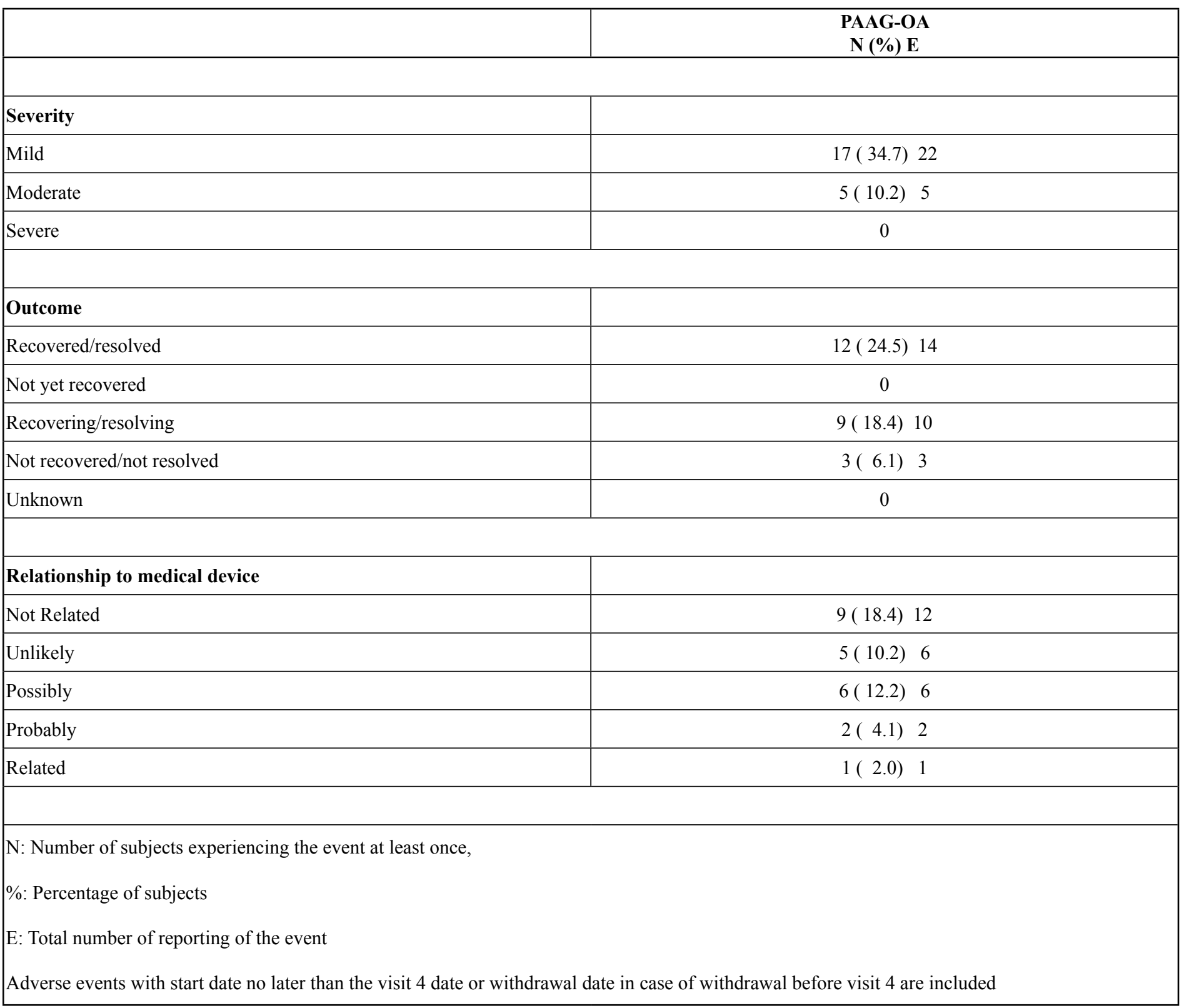

Table 4: Adverse events - 26 weeks.

\begin{tabular}{|c|c|}
\hline $\begin{array}{l}\text { System organ class } \\
\text { Preferred term }\end{array}$ & $\begin{array}{c}\text { PAAG-OA } \\
\text { N (\%) E }\end{array}$ \\
\hline ITT analysis set $(\mathrm{N} \%)$ & $49(100.0)$ \\
\hline Any adverse events & $20(40.8) 27$ \\
\hline
\end{tabular}




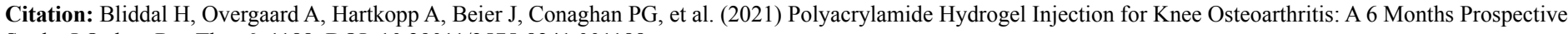
Study. J Orthop Res Ther 6: 1188. DOI: 10.29011/2575-8241.001188

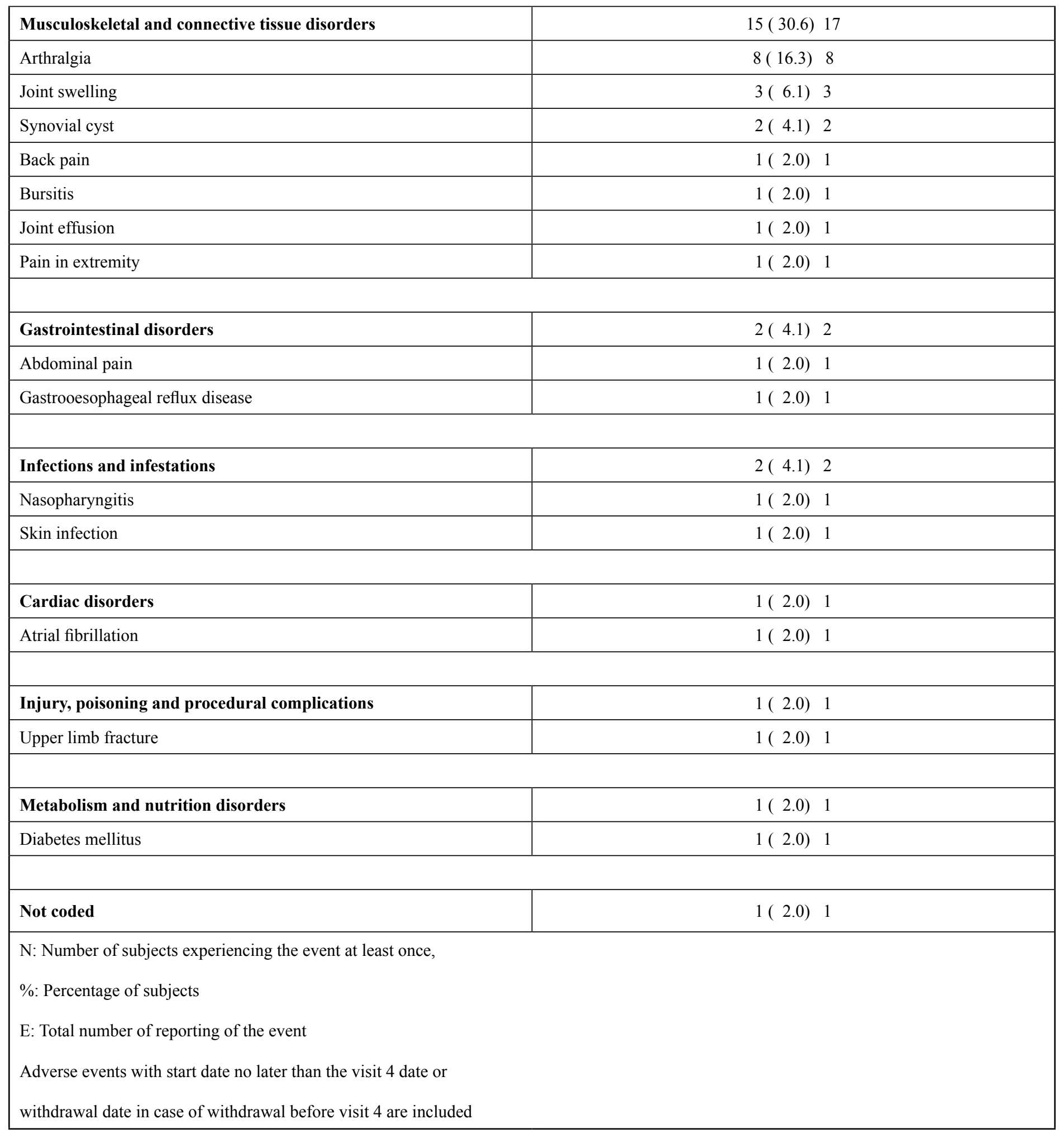

Table 5: Treatment emergent adverse events by system organ class and preferred term -26 weeks - ITT analysis set. 


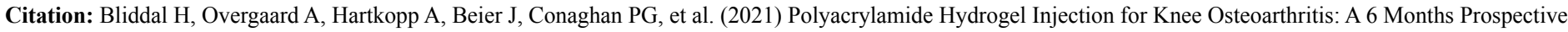
Study. J Orthop Res Ther 6: 1188. DOI: 10.29011/2575-8241.001188

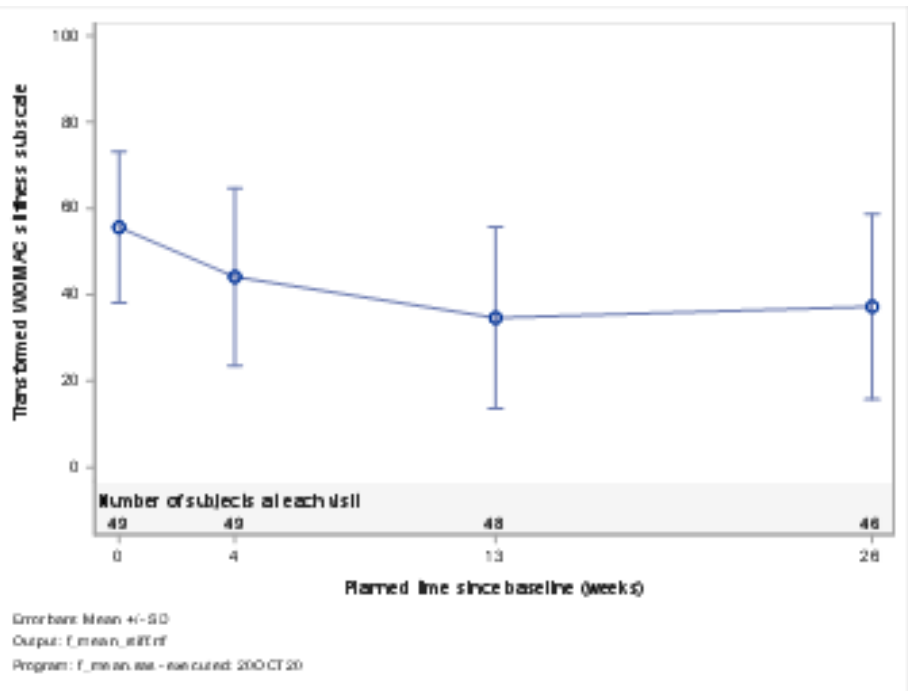

Figure 2: Mean plot of transformed WOMAC stiffness subscale (0-100) - 26 weeks - ITT analysis set.

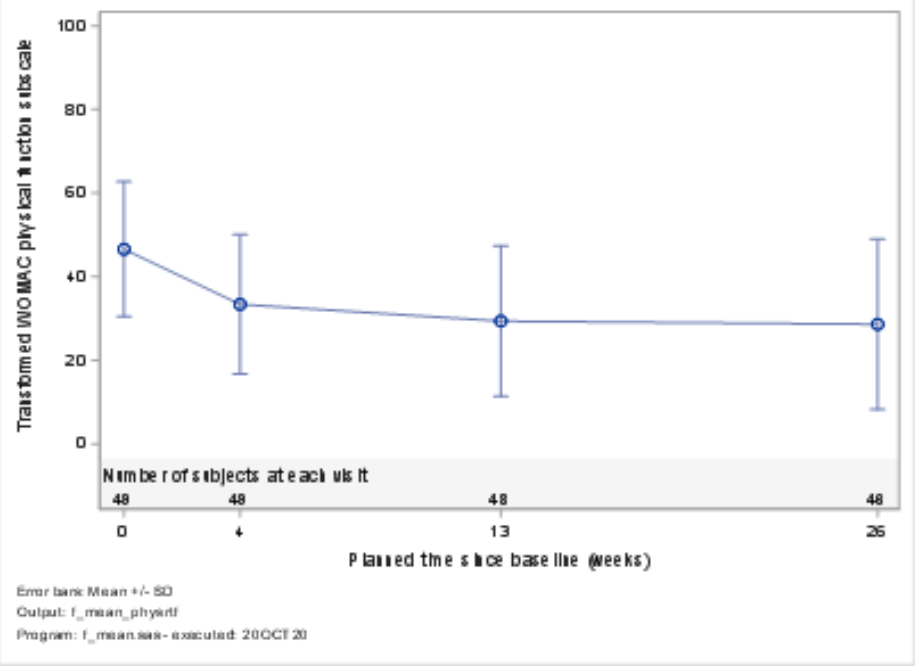

Figure 3: Mean plot of transformed WOMAC physical function subscale (0-100) - 26 weeks.

\section{Discussion}

This open label study in patients with knee OA who received $6 \mathrm{ml}$ PAAG administered as one IA injection showed significant improvement in the WOMAC pain subscale at 4 and 13 weeks which were sustained at 26 weeks. A high percentage of patients achieved MCII at all time points. This was accompanied by improvements in the WOMAC stiffness and function, as well as in the PGA, and two thirds of patients were OMERACT-OARSI responders, demonstrating the efficacy of IAPAAG. PAAG has nonabsorbable, non-biodegradable and non-migratory characteristics.
Upon injection into the joint cavity, the hydrogel integrates with synovial cells which then proliferate, ultimately re-establishing the synovial lining $[7,8]$. As the synovial membrane is highly innervated with nociceptive nerve fibres, this re-establishment of synovium may explain the beneficial effects on pain and other OA symptoms. Although speculative, the action on pain may be via an antiinflammatory effect on the synovium.

The 13 week efficacy outcomes of the single $6 \mathrm{ml}$ injection in this study are similar to the 4 month outcomes of a previously reported observational study where 2 separate injections of $3 \mathrm{ml}$ were tested [4]. Relatively few adverse events were reported in both studies, and all device related AEs were assessed as mild or moderate. In this series, no acute inflammatory adverse reactions was experienced to the injection. However, if such were to occur, the immediate treatment would follow the RICE priniciple (rest, ice, elevation) or, in case of excess joint fluid, aspiration. The 2 reported SAEs were assessed as not related to the device. Thus, the overall findings suggest that the clinical application of PAAG is safe and effective and can be conducted as a single injection, thereby simplifying the treatment strategy. This study is limited by the lack of a control group, which introduces a risk of bias to the results. However, it was designed to detect therapy effects as experienced by the patients and the results are indicative of a considerable benefit following treatment with PAAG, reflecting similar results seen in a previous study $[4,9]$. Nevertheless, a randomised, controlled study is needed to confirm the clinical efficacy and its role in managing patients with knee OA.

\section{Conclusion}

In conclusion, this study in patients with knee OA suggests that $6 \mathrm{ml}$ PAAG can be delivered in a single session with good clinical effects at 26 weeks follow up with no safety concerns raised. The outcomes appear promising but need to be confirmed in a randomized, controlled study.

\section{References}

1. Felson DT, Lawrence RC, Dieppe PA, Hirsch R, Helmick CG, et al. (2000) Osteoarthritis: new insights. Part 1: the disease and its risk factors. Ann Intern Med 133: 635-646.

2. Peat G, McCarney R, Croft $P$ (2001) Knee pain and osteoarthritis in older adults: a review of community burden and current use of primary health care. Ann Rheum Dis 60: 91-97.

3. Tnibar A, Schougaard H, Camitz L, Rasmussen J, Koene M, et al (2015) An international multi-centre prospective study on the efficacy of an intraarticular polyacrylamide hydrogel in horses with osteoarthritis: a 24 months follow-up. Acta Vet Scand 57: 20

4. Henriksen M, Overgaard A, Hartkopp A, Bliddal H (2018) Intra-articular $2.5 \%$ polyacrylamide hydrogel for the treatment of knee osteoarthritis: an observational proof-of-concept cohort study. Clin Exp Rheumatol $36: 1082-1085$ 
Citation: Bliddal H, Overgaard A, Hartkopp A, Beier J, Conaghan PG, et al. (2021) Polyacrylamide Hydrogel Injection for Knee Osteoarthritis: A 6 Months Prospective Study. J Orthop Res Ther 6: 1188. DOI: 10.29011/2575-8241.001188

5. Pham T, Van Der Heijde D, Lassere M, Altman RD, Anderson JJ, et al. (2003) Outcome variables for osteoarthritis clinical trials: The OMERACT-OARSI set of responder criteria. J Rheumatol 30: 16481654.

6. Bellamy N, Hochberg $M$, Tubach $F$, Martin-Mola $\mathrm{E}$, Awada $\mathrm{H}$, et al. (2015) Development of multinational definitions of minimal clinically important improvement and patient acceptable symptomatic state in osteoarthritis. Arthritis Care Res (Hoboken) 67: 972-980.

7. Christensen L, Camitz L, Illigen KE, Hansen M, Sarvaa R, et al. (2016) Synovial incorporation of polyacrylamide hydrogel after injection into normal and osteoarthritic animal joints. Osteoarthritis Cartilage 24: 1999-2002.
8. Tnibar A, Persson AB, Jensen HE (2017) Mechanisms of Action of an Intraarticular 2.5\%Polyacrylamide Hydrogel (Arthramid Vet) in a Goat Model of osteoarthritis: Preliminary Observations. SM Journal of Biomedical Engineering 3.

9. Overgaard A, Bliddal H, Henriksen M (2019) Safety of Intra-Articular Polyacrylamide Hydrogel for the Treatment of Knee Osteoarthritis Symptoms: A Retrospective Case Series. Clin Ortho Adv Res J: COARJ-100001. 